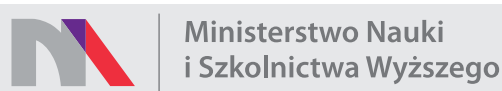

Digitalizacja archiwalnych numerów czasopisma naukowego Analecta Cracoviensia 1-24 (1969-1992) i ich publikacja w otwartym dostępie - zadanie finansowane w ramach umowy 672/P-DUN/2017 ze środków Ministra Nauki i Szkolnictwa Wyższego przeznaczonych na działalność upowszechniającą naukę

\title{
ADAM STEFAN SAPIEHA Métropolitain de Cracovie
}

Il était inconnu à la société de Cracovie, quand le 3 mars 1912, il prenait en possession la Cathédrale du Wawel. Fils du ,prince rouge" Adam Sapicha et d'Hedvige, née Sanguszko, il vit le jour le 14 mai 1867 à Krasiczyn près de Przemyśl. Il fit ses études secondaires à Lwów, et ne fit que passer par Cracovie comme étudiant de la Faculté de Droit à l'Université Jagellonne. Lwów était son diocèse maternel et il y revint à deux reprises: la première fois après avoir terminé ses études théologiques à Innsbruck en Autriche (1893), et ensuite après l'obtention du doctorat en Droit Canon à Rome en 1895. Le Pape Pie X, en 1905, l'appela à ses côtés, lui confiant la charge de chambellan. Un jour, certainement, quelque historien décrira les faits et gestes de notre grand Métropolitain pendant cette période auprès d'un pape qui devait être canonisé - et son rôle très actif aussi bien dans la diplomatie papale, que dans la Commission à laquelle le pape avait confié la codification du Droit Canon de l'Eglise latine.

Adam Sapieha n'était certes pas le seul à se trouver dans les rangs du clergé polonais à Rome, et cependant c'est lui que Joseph Sarto, fils de paysan, ne portant aucune sympathie spéciale aux descendants des grandes familles, a nommé au siège épiscopal de Cracovie, vacant après la mort du cardinal Puzyna, lui conférant lui-même le sacre dans la Chapelle Sixtine (le 17 décembre 1911).

Adam Sapieha rentra dans la capitale de son diocèse au son des fanfares et des cloches de la ville. Pourtant le milieu intellectuel de Cracovie, aux tendances démocratiques et radicales, observait, non sans méfiance, ce nouveau dignitaire qui succédait au peu aimé prince Puzyna, avec lui aussi une mitre princière dans ses armoiries. Il prenait possession de son diocèse à la force de l'âge, après un séjour de quelques années à la Cour papale parmi les grands diplomates et les grands savants. Mais 
il avait aussi à son compte une certaine expérience, acquise dans son pays, où il avait été vicaire de campagne, et ensuite modérateur au Grand Séminaire de Lwów, où en tant que chanoine il avait pu se mettre au courant des affaires du diocèse. A cette dernière fonction on avait ajouté celle d'éducateur de la jeunesse ouvrière de Lwów.

A Cracovie, on ne savait rien de ces choses. Cependant la première lettre pastorale fit savoir aux prêtres et aux fidèles du diocèse que le nouvel évêque allait exiger des prêtres un renouveau intérieur, une intensifícation de l'action pastorale, et le détachement du bien-être matériel. Mais à peine voulut-il commencer ses visites pastorales pour porter aux prêtres et aux fidèles le service de sa parole et de son exemple, que vinrent les années de la première guerre mondiale. C'est alors que, face à la misère et aux malheurs qui tombèrent sur toute la terre polonaise, l'évêque de Cracovie s'enflamma d'une énergie remarquable. Lui, dont la constitution physique était si frêle, travaillait pour deux et pour trois, pour apporter aux milliers de malheureux un soulagement spirituel et une aide matérielle. Au-delà des fronts mouraient des pères et des mères laissant de petits enfants abandonnés. Tout le pays était ravagé par des épidémies de typhus, de variole, de tuberculose, de dysenterie. D'innombrables malades réclamaient des médicaments, de la nourriture, des vêtements. La misère également touchait de plus en plus les habitants mêmes de Cracovie.

Les autorités de la monarchie habsbourgienne ne s'inquiétaient guère de cet état de choses. Leur préoccupation principale était de maintenir les lignes des fronts de guerre, dont le déplacement laissait ruines et déstructions. Tout resta à la charge de la société polonaise, que commençait également à ronger la spéculation, l'égoïsme et l'indifférence aux souffrances des autres. La solidarité des hommes loyaux et nobles était ébréchée par les divergences dans les „orientations politiques”.

L'énormité et le nombre des tâches à accomplir exigeait une planification prévoyante de l'aide à apporter, une organisation solide du travail à faire, une mobilisation entière des moyens financiers et matériels, et surtout une main forte dont personne ne contesterait l'autorité et qui gagnerait la confiance de toutes les couches sociales. Adam Stefan Sapieha prit ces tâches en charge. Avec l'aide d'hommes expérimentés il sut rassembler autour de lui un groupe de personnes généreuses, auxquelles il communiqua son énergie et qu'il enflamma du désir de se consacrer aux autres d'une façon désintéressée au nom des principes chrétiens de la miséricorde, si bien connus mais si mal pratiqués. Sous sa présidence, s'organisa un comité épiscopal (appelé populairement KBK Komitet Książęco-Biskupi = Comité Princier Episcopal). Sa présidence fut loin d'être une simple formalité. 
La plus grande préoccupation de son coeur ardent allait aux enfants. Très vite, il fonda deux établissements, qui subsistèrent après la guerre, et qui peu à peu furent pris en charge par l'Université Jagellonne. Ce furent deux magnifiques monuments de l'action entreprise par l'évéque de Cracovie: à Witkowice près de Cracovie, une maison de santé four enfants malades de conjonctivite granuleuse, et à Bystre (Zakopane) un sanatorium pour enfants malades de tuberculose.

Les habitants de Cracovie savaient ce qu'ils devaient à leur évéque et se sont sincèrement attachés à sa personne. Ils étaient fiers d'avoir pour évêque ce grand aumônier — plein de miséricorde - qu'était Adam Stefan Sapieha.

Dans la Pologne restaurée - étant dès l'année 1925 archevêque de la Métropole de Cracovie érigée par suite du Concordat - il consacra une attention spéciale aux questions pastorales et aux problèmes d'éducation. Il voyait venir des temps nouveaux qui exigeraient de nouvelles méthodes de travail, pour que les principes de la moralité chrétienne ne restent pas lettre morte, mais pénètrent la vie de tous les fidèles de la société polonaise. Dès son arrivée au siège épiscopal de Cracovie, il concentra son attention sur la vie des séminaristes et des prêtres. Il voulait qu'ils soient à même de comprendre la nouvelle réalité polonaise et soient solidement préparés à la charge de guides de la vie intérieure des fidèles pour les conduire par un exemple véritable de piété, de droiture et de générosité. Il se rendait compte que les conditions de plus en plus complexes de la vie sociale, économique et politique exigeaient également une préparation solide des prêtres aussi bien dans le domaine de la théologie et de la philosophie que dans celui des sciences sociales. Aussi entoura-t-il d'un soin particulier la Faculté Théologique de l'Université Jagellonne, qui devint le centre de la formation du clergé non seulement de Cracovie mais aussi de Silésie et de Częstochowa. L'Université de Cracovie - par les séminaristes qui venaient du diocèse de Katowice devait à nouveau fortifier le sentiment polonais et l'amour de la patrie sur ces terrains qui avaient été pris par les Prussiens et forcés par eux à devenir allemands. Les trois Grands Séminaires, établis à Cracovie, ne voulaient pas être uniquement une école de vie ascétique. Les jeunes prêtres qui devaient en sortir, y étaient préparés au ministère pastoral à tout point de vue, aussi bien intellectuellement que physiquement. La gymnastique et le sport figuraient au programme. Les jeunes séminaristes, grâce à leur adhésion au Bratniak (genre de Fraternité et d'entraide pour la jeunesse universitaire) s'entrainaient à la vie sociale et prenaient connaissance des courants de pensée de la jeune génération de la Pologne restaurée. Des professeurs civils (parmi eux se trouvait aussi Teodor Marchlewski, un des recteurs de l'Université Jagellonne 
après la guerre), donnaient des cours d'initiation aux problèmes des sciences biologiques et sociales comme aussi aux questions de la politique confessionnelle et de la vie de l'Etat. Il envoyait de jeunes prêtres doués faire des études spécialisées non seulement à Rome, où grâce à ses démarches l'ancien hospice Saint-Stanislas était rendu à sa première fonction, mais aussi en Autriche (Innsbruck), en France (Strasbourg), et en Belgique (Louvain et Liège) où lui-même pendant un certain temps avait poursuivi des études.

Un courant de saine nouveauté pénétrait également les rangs du clergé plus agé du diocèse de Cracovie. Un échange d'idées avait lieu non seulement à l'Institut d'Etudes Catholiques, fondé par ces prêtres sur le conseil de l'xcellent philosophe Konstanty Michalski, mais aussi dans des rencontres privées.

Le Métropolitain de Cracovie entourait d'un soin particulier l'Action Catholique. Un grand édifice, construit de par son initiative au coin des rues Straszewski et Zwierzyniecka, devint le centre d'une intense action intellectuelle, atteignait et Cracovie et tout le diocèse. Dans les salles de lectures, dites „,salle dorée”, et „salle bleue”, - exceptionnellement pour des académies plus importantes dans la salle du cinéma „Swit" qui est devenu aujourd'hui la „Philharmonie” de Cracovie - se rassemblaient des foules de Cracoviens pour écouter des cours qui donnaient le point de vue chrétien sur des questions économiques, sociales et culturelles, et prendre part à des discussions vivantes. Par principe, on ne traitait pas de questions politiques actuelles, bien que le Métropolitain s'y intéressât très vivement.

Ennemi du germanisme, il était pendant la première guerre l'adversaire décidé de la solution autrichienne de la question polonaise. Fidèle au principe, selon lequel le pasteur des fidèles devait s'abstenir de prendre la parole dans des questions de politique - il ne proclamait pas lui-même publiquement ses opinions. Il laissait ce soin à l'organe cracovien de l'intelligence catholique, „Glos Narodu” (La Voix de la Nation), avec lequel collaborait alors très étroitement Stanisław Kutrzeba, professeur de l'Université Jagellonne, qui devait devenir secrétaire général et ensuite président de l'Académie Polonaise des Sciences. Par le „Głos Narodu" les habitants de Cracovie savaient exactement quelle était l'opi nion de leur évêque sur ces questions.

- Au moment du coup d'Etat de mai (1926), tout le monde savait qu'il reprouvait tout abus d'autorité et toute illégitimité, comme plus tard il devait condamner l'ignominie de Brześć, l'emprisonnement et le traitement cruel des hommes politiques, encore que les opinions de bien d'entre eux étaient pour lui inacceptables. On parla de nouveau beaucoup de lui, quand en 1937 il fit transférer le corps de Piłsudski dans la crypte pré- 
paré à cet effet sous la Tour des Cloches d'Argent dans la Cathédrale du Wawel. Cette décision ne découlait nullement d'une volonté quelconque de démonstration politique. Il avait toujours protesté contre les innombrables allées et venues, souvent obligatoires, d'une masse de jeunes étudiants qui faisaient de la cathédrale, maison de Dieu, un vestibule malpropre de la crypte. Comme son intervention auprès du président Mościcki et son exigence de respect pour la sainteté de ce lieu de prière s'était avérée inefficace, de sa propre initiative, il donna l'ordre de transférer le sarcophage dans un endroit qui avait été depuis longtemps choisi en accord avec les autorités civiles. Autour de cet acte - qui met en pleine lumière la droiture et la force de décision du Métropolitain de Cracovie - éclata un orage, auquel il sut tenir tête et qu'il subit avec dignité, fort de sa foi dans la justesse de sa décision.

Les échos ne s'en étaient pas encore tus, que vinrent les jours longs et pénibles de l'occupation hitlérienne. Parmi ceux qui connaissaient le Métropolitain de Cracovie, personne ne s'étonna, au moment des jours cruels de septembre - alors que le Président de la République avec tous les membres du gouvernement, avec le Nonce et le Cardinal Hlond quittaient les frontières du pays - de ce que lui resta au milieu de ses diocésains. Il resta pour partager leur sort, pour les défendre autant qu'il pourra se faire.

Les hitlériens, avec Frank, petit roi du Gouvernement général, en tête, savaient bien que le frêle et menu dignitaire de l'Eglise, aux yeux noirs et perçants sous une chevelure grisonnante, n'était pas seulement un administrateur d'office. Ils savaient que vers le Métropolitain de Cracovie étaient tournés les yeux de toute la nation, et que son inflexibilité était le soutien de celle de la Pologne, de cette nation qui souffrait et luttait, mais ne doutait jamais de la victoire de la bonne cause et de la reconquête de la souveraineté.

Pendant la période si pénible de l'occupation, il vivait de la même vie que tous les Polonais. Aussi incertain que les autres du lendemain, aussi éprouvé par le sort de ses proches qui tombaient aux mains de la Gestapo, touché par le malheur qui frappait les professeurs de l'Université Jagellonne dont un grand nombre devait périr dans les camps de concentration, touché par le sort du clergé paroissial que la Gestapo décimait peu à peu, par le sort de toute la nation polonaise qui chaque jour perdait son sang, par le sort de tous les compatriotes enfermés dans les camps de prisonniers ou déportés par les hitlériens dans les camps de travail.

Bientôt, peut-être, un historien compétent pourra d'une façon dé taillée et objective décrire cette lutte incessante du grand Métropolitain avec les autorités hitlériennes, pour au moins adoucir leurs mesures 
inhumaines et leurs abus continuels de pouvoir. Pour ne citer qu'un exemple de ses grands soucis, je dois rappeler la préoccupation avec laquelle il s'intéressait au sort des milliers de déportés aux travaux forcés dans le Reich. Il savait qu'ils étaient privés de tout secours religieux, condamnés à la démoralisation, que des hommes et des femmes qui faisaient connaissance dans ce milieu hostile, ne pouvaient se marier, que nombreux étaient ceux qui mouraient sans l'assistance du prêtre. Il avait voulu envoyer dans le Reich des prêtres polonais pour s'occuper des déportés. Le Cardinal Bertram constatait que cette proposition était impossible à réaliser à cause du refus des autorités civiles. Ses interventions au Vatican furent tout aussi inefficaces à cause de l'attitude des autorités allemandes et de la petitesse du clergé allemand qui n'osait informer les Polonais de la possibilité de mariage sacramentel sans l'assistance du ministre propre.

De même qu'il avait été, pendant la première querre, l'âme du KBK, ainsi, pendant la seconde guerre mondiale, sans en être le chef formel, il était à la tête du Conseil Administratif Principal, toléré par les Allemands, et de la section cracovienne de la Croix Rouge Polonaise, étant lui-même l'auteur de nombreuses initiatives.

Face à l'ennemi, il était intransigeant et sans peur. Toute la société polonaise le savait bien, et ceux qui demeuraient dans le pays, et ceux que le sort avait jeté en dehors des frontières de la patrie. Pas une fois, Adam Sapieha ne franchit le seuil de sa Cathédrale du Wawel, tant que Frank par sa présence déshonorait les chambres royales du Château.

Arriva enfin le jour attendu avec impatience par tous ceux auxquels Dieu avait donné de survivre. L'armée russe porta à l'armée allemande un coup mortel. Mais sur nos terres la paix n'était pas encore faite. Une lutte s'engagea pour une nouvelle structure de l'Etat, où il ne manqua ni de coups de feu, ni de sang versé.

De nouveau, les yeux de la société se tournèrent vers la personne du Métropolitain de Cracovie. En toute responsabilité, je peux dire que le Parti Populaire Polonais avait émis une suggestion sollicitant le Cardinal à engager son autorité dans cette lutte politique. Le Métropolitain de Cracovie la rejetta sans hésiter. Il déclara que les autorités ecclésiastiques ne pouvaient prendre part à des luttes politiques. Il appartient à l'Eglise de veiller à ce que les fidèles observent les commendements de Dieu, puissent approfondir leur vie intérieure, et vivent selon les principes de la morale chrétienne, telle que l'enseigne l'Eglise. Tant que l'Eglise peut en toute liberté remplir sa fonction pastorale, tant qu'est respectée la liberté de conscience et la liberté des pratiques religieuses, tant qu'est sauvegardée la liberté de l'éducation religieuse et l'observance des principes de la morale chrétienne - l'autorité civile est et 
sera respectée par le clergé à tous les dégrés de la hierarchie, et les fidèles seront invités à la respecter également.

Dans quelle mesure l'attitude du Métropolitain de Cracovie était le fruit de sa décision personnelle, ou bien l'effet des opinions de l'Episcopat Polonais - qui certes n'étaient pas sans influence - il m'est imposible de le dire.

Mais il lui fallait se remettre à rebâtir la vie religieuse dans l'archidiocèse de Cracovie avec toujours plus d'effort durant les dernières années de sa vie, dont les mérites furent couronnés par le chapeau cardinalice que l'Archevêque Adam Sapieha reçut des mains du pape en 1946. Cette distinction lui revenait depuis longtemps, non seulement selon la tradition du siège épiscopal qu'il occupait, mais surtout à cause de ses mérites personnels. L'opinion publique générale - l'histoire dira si elle est juste - impute ce retard au conflit qui survint entre lui et le nonce apostolique, Mgr Achille Ratti, futur pape Pie XI, à propos du retour de la Silésie à la Pologne. C'est pourquoi l'attribution de la dignité cardinalice au Métropolitain de Cracovie fut considérée comme un fait naturel qu'il aurait fallu accomplir depuis longtemps.

La pourpre ne le changea en rien. Il continua à se montrer dans les rues de Cracovie, quoique plus rarement et d'un pas plus alourdi, en soutane noire bordée d'un filet de pourpre très discret, avec son vieux chapeau noir aux larges bords. Ses jours étaient comptés, et la fin s'approchait implacable. Il s'éteignit le 23 juillet 1951 . Son enterrement fut digne de la sépulture d'un roi. Ce fut une manifestation grandiose des sentiments de tout le clergé polonais et de toute la société du diocèse de Cracovie. Les témoins de cette journée mémorable et de ces foules en prière qui accompagnaient la dépouille mortelle du Cardinal de la crypte au Wawel, ne pourront l'oublier. Tant étaient grands l'amour et la vénération de toute la société catholique de notre pays pour le grand Métropolitain de Cracovie. 